\title{
Erratum to: Amelioration of autoimmune arthritis by adoptive transfer of Foxp3-expressing regulatory $B$ cells is associated with the Treg/Th17 cell balance
}

Mi-Kyung Park ${ }^{1 \dagger}$, Young Ok Jung ${ }^{2 \dagger}$, Seon-Yeong Lee ${ }^{1 \dagger}$, Seung Hoon Lee ${ }^{1}$, Yu-Jung Heo ${ }^{1}$, Eun-Kyung Kim', Hye-Jwa Oh ${ }^{1}$, Young-Mee Moon ${ }^{1}$, Hye-Jin Son ${ }^{1}$, Min-Jung Park' , Sung-Hwan Park³, Ho-Youn Kim³ ${ }^{3}$ Mi-La Cho ${ }^{\text {** }}$ and Jun-Ki Min ${ }^{4,5^{*}}$

\section{Erratum to: J Transl Med (2016) 14:191 DOI 10.1186/s12967-016-0940-7}

Unfortunately, the original version of this article [1] contained errors. The author's names were incorrectly marked up and display incorrectly on PubMed. Additionally, the funding information was incorrect. The correct author list and funding section can be found below. The author names and funding section have been corrected in the original article [1] and is also included correctly in this erratum.

\footnotetext{
Author details

${ }^{1}$ The Rheumatism Research Center, Catholic Research Institute of Medical Science, The Catholic University of Korea, 505 Banpo-dong, Seocho-gu, Seoul 137-040, South Korea. ${ }^{2}$ Division of Rheumatology, Department of Internal Medicine, Hallym University Kang-Nam Sacred Heart Hospital, Seoul, South Korea. ${ }^{3}$ Division of Rheumatology, Department of Internal Medicine, College of Medicine, The Catholic University of Korea, Seoul, South Korea. ${ }^{4}$ Bucheon St. Mary's Hospital, Division of Rheumatology, Department of Internal Medicine, College of Medicine, The Catholic University of Korea, 327 Sosa-ro, Wonmi-gu, Bucheon, Gyeonggi-do 420-717, South Korea. ${ }^{5}$ Division of Rheumatology, Department of Internal Medicine, College of Medicine, Holy Family Hospital, Rheumatism Research Center (RhRC), Catholic Research Institute of Medical Science, The Catholic University of Korea, Seoul, South Korea.
}

\begin{abstract}
Funding
This study was supported by a grant of the Korean Health Technology R\&D Project, Ministry for Health \& Welfare, Republic of Korea (HI14C3417). This study was supported by a grant of the Korean Health Technology R\&D Project, Ministry for Health \& Welfare, Republic of Korea (HI14C1851). This research was supported by Basic Science Research Program through the National Research Foundation of Korea (NRF) funded by the Ministry of Education (NRF-2015R1D1A1A01057072).
\end{abstract}

Published online: 26 July 2016

The online version of the original article can be found under doi:10.1186/s12967-016-0940-7.

\section{Reference}

1. Park M-K, Jung YO, Lee S-Y, Lee SH, Heo Y-J, Kim E-K, Oh H-J, Moon Y-M, Son H-J, Park M-J, Park S-H, Kim H-Y, Cho M-L, Min J-K. Amelioration of autoimmune arthritis by adoptive transfer of Foxp3-expressing regulatory B cells is associated with the Treg/Th17 cell balance. J Transl Med. 2016;14:191. doi:10.1186/s12967-016-0940-7.

*Correspondence: iammila@catholic.ac.kr; rmin6403@hanmail.net

${ }^{\dagger}$ Mi-Kyung Park, Young Ok Jung and Seon-Yeong Lee contributed equally to this work

${ }^{\ddagger} \mathrm{Mi}$-La Cho and Jun-Ki Min contributed equally to this work

${ }^{1}$ The Rheumatism Research Center, Catholic Research Institute of Medical Science, The Catholic University of Korea, 505 Banpo-dong, Seocho-gu, Seoul 137-040, South Korea

${ }^{4}$ Bucheon St. Mary's Hospital, Division of Rheumatology, Department of Internal Medicine, College of Medicine, The Catholic University of Korea, 327 Sosa-ro, Wonmi-gu, Bucheon, Gyeonggi-do 420-717, South Korea

Full list of author information is available at the end of the article 